OPEN ACCESS

Edited by:

Min Tang,

University of Applied Management,

Germany

Reviewed by:

Chihwen Wu,

National Chung Hsing University,

Taiwan

Mashura Shammi,

Jahangirnagar University, Bangladesh

${ }^{*}$ Correspondence:

Quanlei Yu

yulei19881987@mail.ccnu.edu.cn

Zhiijin Zhou

zhouzj@mail.ccnu.edu.cn

Qingbai Zhao

zqbznr@mail.ccnu.edu.cn

tThese authors have contributed equally to this work

Specialty section:

This article was submitted to

Organizational Psychology,

a section of the journal

Frontiers in Psychology

Received: 29 August 2020 Accepted: 07 May 2021

Published: 14 June 2021

Citation:

Wu X, Wang Z, Zhang $H$, Yuan $P$, Yu Q, Zhou Z and Zhao Q (2021)

Effects of Internet Language Related

to COVID-19 on Mental Health in College Students: The Mediating

Effect of Cognitive Flexibility.

Front. Psychol. 12:600268.

doi: 10.3389/fpsyg.2021.600268

\section{Effects of Internet Language Related to COVID-19 on Mental Health in College Students: The Mediating Effect of Cognitive Flexibility}

\author{
Xingzhe Wu ${ }^{1 \dagger}$, Zhi Wang ${ }^{1,2+}$, Hongpo Zhang ${ }^{1}$, Peiming Yuan ${ }^{1}$, Quanlei Yu ${ }^{1,3 *}$, \\ Zhijin Zhou ${ }^{1 *}$ and Qingbai Zhao ${ }^{1 *}$
}

${ }^{1}$ School of Psychology, Central China Normal University, Wuhan, China, ${ }^{2}$ Mental Health Education and Counseling Center, Youjiang Medical University for Nationalities, Guangxi, China, ${ }^{3}$ Key Laboratory of Adolescent Cyberpsychology and Behavior (CCNU), Ministry of Education, Wuhan, China

During the COVID-19 pandemic, Internet language (INL) has influenced daily life extensively. However, the process by which INL influences people's psychology and behavior is unclear. This study explored the effects of INL on mental health (anxiety and depression). A pilot study was conducted to develop a qualified scale for INL related to COVID-19 (CINL) in college students using an online questionnaire. The CINL scale was found to have two dimensions: frequency and comprehension, as well as good reliability and validity. A formal study explored the mediating effect of cognitive flexibility on the relationship between CINL and mental health. The results showed that CINL positively predicted mental health when it was mediated by cognitive flexibility. These results not only provide a new perspective on understanding the effects of cyber behavior on human mental health from a positive perspective, but also provide practitioners with new insights for interventions on college students' mental health.

Keywords: Internet language, cognitive flexibility, anxiety, depression, COVID-19

\section{INTRODUCTION}

At the beginning of 2020, COVID-19 broke out worldwide, and as of April 18, 2021, the cumulative number of confirmed cases and deaths globally had reached 140,578,995 and 3,010,453, respectively (World Health Organization, 2021). COVID-19 has spread rapidly across the world and is a source of ongoing concern. It is worth noting that stress from the risks associated with the COVID-19 pandemic - social alienation, the implementation of isolation measures, and the consequences of the disease - significantly impact people's mental health and increase the risk of mental health problems (Vahia et al., 2020). People who are diagnosed with or suspected of being infected with COVID-19 experience negative emotional and behavioral reactions, such as fear, loneliness, anxiety, and insomnia (Ornell et al., 2020). In addition, COVID-19 also has a significant negative impact on people who are not infected. A recent study conducted in China demonstrated that about $53.8 \%$ of the respondents began to suffer from a moderate or severe psychological disorder during the pandemic. In particular, $16.5 \%$ reported experiencing moderate 
to severe depressive symptoms, $28.8 \%$ reported experiencing moderate to severe anxiety symptoms, and $8.1 \%$ experienced moderate to severe stress (Wang et al., 2020).

A vast body of Internet language (INL) has emerged in the context of the COVID-19 pandemic. INL in China refers to a language generated or applied in online communication and is composed of Chinese and English letters, punctuation marks, symbols, pictures, characters, and so on ( $\mathrm{Wu}, 2004$; Du, 2007). Generally, these combinations express special meanings in online communication. Previous studies suggest that Chinese INL has many types, including those that parse Chinese characters (e.g., combination of two Chinese characters “马” meaning "bow," and “虽” meaning “though” into a new Chinese character “强”, meaning "strong"), stylized numbers (e.g., the number 94 sounds like "jiu shi" in Mandarin, which means confirmation), and so on. With the rapid development of the Internet, the forms and content of INL are becoming increasingly diversified. In the context of COVID-19, some common words have been given new usages or interpretations. For example, in the past, the idiom “du (度) ri (日) ru (如) nian (年),” which means “getting through a day is like getting through a year," is used to describe the experience of going through a difficult time; however, netizens have given this idiom a new meaning: "during the pandemic, individuals do not need to work, and they can relax as they would on the lunar New Year holiday." Another phrase that has emerged is: "before the pandemic, we were afraid to encounter ghosts; however, during the pandemic, we are afraid to encounter people." However, it is not sufficient to merely summarize all the types of INL. Many studies have demonstrated that individuals who browse or use INL experience positive emotions that are often accompanied by insights (Ma et al., 2012; Shen et al., 2017). Therefore, INL is generally characterized as being humorous. Furthermore, understanding INL requires users to adopt unusual perspectives to interpret INL's novel meanings (Zhou, 2010; Xu, 2015); thus, novelty is another characteristic of INL.

Generally, INL is updated quickly based on current events. Whether this refers to events in daily life or during the COVID19 pandemic, INL has been found to influence study, work, and life. Among these, mental health, such as anxiety and depression, is also affected. On the one hand, because INL is usually humorous in tone, it can trigger positive emotions (happy, relaxed, etc.) that can promote mental health (Guo and Wang, 2007; Zhao et al., 2010). In addition, the humor of INL has an entertainment function, so the imitation and use of INL can easily close interpersonal distance, increase interpersonal attraction, and further satisfy the need for belonging (Yao and Zhou, 2019), which could also impact mental health. Therefore, we hypothesized that INL can directly improve the mental health of netizens during COVID-19.

On the other hand, because of the novelty of INL, which can help individuals to view things from multiple perspectives and improve their understanding of the same thing, INL could also improve individuals' cognitive flexibility, which is defined as the ability of human beings to change cognitive processing strategies in response to new and unexpected situations in their environment (Canas et al., 2003; Al-Mosaiwi and Johnstone, 2018). The effect of novel stimuli on cognitive flexibility has been supported by many previous empirical studies (Barceló et al., 2002; Jowkar-Baniani and Schmuckler, 2014).

Previous studies have found that individuals with high cognitive flexibility are more likely to consider a situation from a different point of view and switch their ideas smoothly, resulting in individuals with high cognitive flexibility and higher levels of life satisfaction, self-esteem, and mental health (Kim and Omizo, 2005; Koesten et al., 2009; Windle, 2010; Jen et al., 2018; Odacı and Çikrikci, 2018). Furthermore, individuals with high cognitive flexibility are more likely to meet the requirements of the environment, efficiently cope with difficult situations, adapt their thoughts and behaviors, and be good at problem solving. This leads to a higher likelihood of success in settling individual internal conflicts and clashes with the outside world, and in establishing a good balance with the outside world (Tchanturia et al., 2004; Hasselbalch et al., 2012; Murphy et al., 2012; Brewster et al., 2013; Mehri and Bakhtiarpoor, 2016; Perpiñá et al., 2016; Jahja et al., 2017; Edwards, 2019). Therefore, this study further hypothesized that INL may have an indirect effect on mental health through cognitive flexibility during COVID-19.

In other words, while people are at risk of mental health issues during the pandemic, INL may affect the mental health of netizens through direct and indirect paths. Therefore, this study synthetically assumed that INL promotes netizens' mental health during COVID-19, which may be partially mediated by cognitive flexibility.

To explore the effect of INL on mental health during the COVID-19 pandemic, a pilot study was conducted to develop a scale for INL in relation to COVID-19 (CINL). A cross-sectional survey was conducted to explore the mediating effect of cognitive flexibility on the relationship between CINL and mental health. To obtain sufficient and suitable data, this study developed an online questionnaire for data collection. Previous studies have found that mental health is influenced by participants' gender (Zhang and Yu, 2004; Feng and Zhang, 2005; He, 2014), age (Zhang et al., 2011), physical health status (Dong et al., 2012; Lu and Wang, 2015), family economic status (Hudson, 2005; Hu et al., 2014), and physical activity (Swann et al., 2018; Zheng et al., 2020). To eliminate the interference of these variables, this study included these variables as control variables.

\section{PILOT STUDY: DEVELOPING A SCALE OF INL RELATED TO COVID-19}

Although previous studies have focused on the use of INL (Liu et al., 2017, 2019; Zhao et al., 2017; Zhang et al., 2019), there are no qualified tools to measure INL. Furthermore, a scale for INL related to COVID-19 requires specificity and effectiveness. Therefore, to explore the effect of CINL on mental health, it was necessary to develop a scale for CINL with good reliability and validity.

\section{Theoretical Construction}

As suggested by previous studies (Yang et al., 2006; Yang and Li, 2007; Yu et al., 2017), frequency are often used as indicators to measure behavior. This study adopted frequency as one 
dimension of CINL. As the humorous or pleasant emotions aroused by INL are usually transient rather than lasting, people may need to use INL over sufficient duration and frequency to maintain a good state of mind to eliminate anxiety and depression. Furthermore, INL is different from other types of Internet communication; it is not only a behavior but also a cognitive process. The effect of INL may also depend on how it is understood (Huang et al., 2013). Take the idiom “du (度) ri (日) ru (如) nian (年)” as an example. If netizens understood this idiom in its original rather than its novel meaning, the INL would have no effect. It is remarkable that novelty is relative but not permanent; it may have been amazing for people to travel by plane, but now it is normal to fly around the world. Therefore, this study considers comprehension as another dimension of INL.

Based on this, the research team, which included four college teachers and three psychology graduates, developed a scale that included items in two dimensions. The frequency dimension consisted of three items (e.g., "The number of times I browse words, sentences, or paragraphs with INL related to COVID-19") and the comprehension dimension consisted of four items (e.g., "In my life, I will try my best to use these words, sentences, or paragraphs with INL related to COVID-19"). This pilot study further established the validity and reliability of the CINL scale.

\section{Structural Validation Participant}

In total, 526 university students were recruited from a medical college in Guangxi Zhuang Autonomous Region (GZAR), China. To eliminate the possible effects of confounding variables, 116 participants were excluded because they had provided very similar responses in the survey. Finally, valid data from 416 participants were obtained (79.09\% of the original sample size). The sample $(N=416)$ was randomly divided into two subgroups. Data from subgroup 1 were subjected to an exploratory factor analysis; it included data from 219 participants whose ages ranged from 18 to 23 years old, with an average age of 19.76 years $(S D=1.19)$, of whom 84 were male $(38.40 \%)$. Data from subgroup 2 were subjected to a confirmatory factor analysis, and this subgroup included 197 respondents whose ages ranged from 17 to 31 years old, with an average age of 19.91 years $(S D=1.56)$, of whom 87 were male students (44.20\%).

\section{Validity and Reliability Analysis Discrimination}

An item-total correlation was used to test for discrimination of items, as suggested by classical test theory (CTT) (Lord and Novick, 1968). When the correlation coefficient between the item score and total score was greater than 0.40 , the item was retained. As shown in Table 1, all the item-total correlation coefficients ranged between 0.43 and 0.73 . Therefore, no items were excluded from this study.

TABLE 1 | Correlation coefficient between each item score and total score.

\begin{tabular}{cccccccc}
\hline Item & $\mathbf{1}$ & $\mathbf{2}$ & $\mathbf{3}$ & $\mathbf{4}$ & $\mathbf{5}$ & $\mathbf{6}$ & $\mathbf{7}$ \\
\hline Score & 0.69 & 0.72 & 0.72 & 0.61 & 0.57 & 0.43 & 0.57
\end{tabular}

\section{Exploratory factor analysis (EFA)}

Data from subgroup 1 were subjected to exploratory factor analyses. First, Kaiser-Meyer-Olkin (KMO) and Bartlett sphere tests were conducted, and the results indicated that the data were suitable for factor analysis $(\mathrm{KMO}=0.74$; Bartlett Sphere: $\left.\chi^{2}=573.54, p<0.001\right)$. Subsequently, the principal component analysis method was used to extract factors with an eigenvalue greater than 1, and the maximum variance method was used to rotate the component matrix. Two factors were extracted, and the total contribution rate was $67.64 \%$. According to CTT (Lord and Novick, 1968), items should not load two or more factors, and its factor load should not be less than 0.3; otherwise, the item should be dropped. As shown in Table 2, the sixth item loaded two factors and was excluded. Finally, the remaining six items were retained. The exploratory factor analysis was performed again with six items, as shown in Table 3. After factor rotation, these six items could load on two factors, which explained $73.42 \%$ of the total variation rate. These two factors were consistent with the theoretical construction; thus, this study named the two factors "frequency of use of CINL" and "comprehension of use of CINL."

\section{Confirmatory factor analysis (CFA)}

A confirmatory factor analysis was performed using data from subgroup 2. The macro program Amos was used to conduct a confirmatory factor analysis to test the two-factor model obtained by exploratory factor analysis. The results (Table 4)

TABLE 2 | Results of EFA of the CINL scale before revision.

\begin{tabular}{lcc}
\hline & \multicolumn{2}{c}{ Factor load } \\
\cline { 2 - 3 } & $\mathbf{1}$ & $\mathbf{2}$ \\
\hline $\begin{array}{l}\text { 1. How many time I browse words, sentences or } \\
\text { paragraphs using INL about COVID-19 }\end{array}$ & 0.88 & 0.02 \\
$\begin{array}{l}\text { 2. How much time I spend in browsing words, } \\
\text { sentences or paragraphs using INL about COVID-19 }\end{array}$ & 0.88 & 0.07 \\
$\begin{array}{l}\text { 3. How frequently I browse words, sentences or } \\
\text { paragraphs using INL about COVID-19 }\end{array}$ & 0.89 & 0.12 \\
$\begin{array}{l}\text { 4. I feel happy when I browse words, sentences or } \\
\text { paragraphs using INL about COVID-19 }\end{array}$ & 0.01 & 0.88 \\
$\begin{array}{l}\text { 5. I feel engaged when I browse words, sentences or } \\
\text { paragraphs using INL about COVID-19 } \\
\text { 6. I feel impressed when I browse words, sentences or } \\
\text { paragraphs using INL about COVID-19 } \\
\begin{array}{l}\text { 7. In daily life, I will try my best to use INL words, } \\
\text { sentences or paragraphs about COVID-19 }\end{array}\end{array}$ & 0.05 & 0.86 \\
\hline
\end{tabular}

TABLE 3 | Results of EFA of the CINL Scale after revision.

\begin{tabular}{lccccc}
\hline & \multicolumn{2}{c}{ Factor 1 } & & \multicolumn{2}{c}{ Factor 2 } \\
\cline { 2 - 3 } \cline { 5 - 6 } & Item & Loading & & Item & Loading \\
\cline { 5 - 6 } & 1 & 0.88 & & 4 & 0.86 \\
Eigenvalues & 3 & 0.89 & & 5 & 0.87 \\
Cumulative (\%) & 2.40 & 0.90 & & 7 & 0.70 \\
& $39.93 \%$ & $73.42 \%$ & & &
\end{tabular}


TABLE 4 | Results of confirmatory factor analysis of the CINL scale.

\begin{tabular}{lccccccc}
\hline$\chi^{2}$ & df & $\chi^{2} / \mathbf{d f}$ & RMSEA & NFI & RFI & CFI & IFI \\
\hline 11.033 & 8 & 1.38 & 0.044 & 0.98 & 0.96 & 0.99 & 0.99 \\
\hline
\end{tabular}

indicate that the two-factor model of the CINL scale has good structural validity.

\section{Reliability analysis}

Cronbach's alpha and split-half tests were conducted to establish the reliability of the CINL scale. Results showed that the Cronbach's alpha of the frequency and comprehension dimensions were 0.88 and 0.74 , respectively. The split-half reliability coefficients of the two dimensions were 0.86 and 0.60 , respectively.

\section{Discussion}

This pilot study developed a CINL scale that has two dimensions, namely, frequency and comprehension. The results of the validity and reliability analysis met psychometric requirements. However, the reliability of the comprehension dimension of the CINL scale was 0.60 , which is relatively low. This may be because this dimension has fewer items (Angoff, 1953; Friedland and Michael, 1987; Hellman et al., 2006).

The CINL scale is the first tool to comprehensively measure behavior related to INL usage, and provides a theoretical and practical basis for future studies to explore the relationship between INL and human psychology and behaviors.

\section{THE MEDIATING EFFECT OF COGNITIVE FLEXIBILITY BETWEEN CINL AND MENTAL HEALTH}

Based on the results of the pilot study, this study further explored the mediating effect of cognitive flexibility on the relationship between CINL and mental health.

\section{Participants}

The sample was the same as in Study 1.

\section{Measures}

\section{Measurement of CINL}

A self-compiled scale was used to measure CINL. Responses to the items were rated on a 5-point Likert scale, ranging from 1 (strongly disagree) to 5 (totally agree). The sum of the scores was considered an indicator of the influence of CINL. See Study 1 for detailed usage methods.

\section{Cognitive Flexibility}

The cognitive flexibility scale (CFI) (Dennis and Wal, 2010), which was translated into Chinese by Wang et al. (2016), was used to measure participants' cognitive flexibility. This scale is composed of the following dimensions: controllability and choice. The controllability dimension contains 12 items (e.g., "I am good at analyzing and evaluating various situations"), while the choice dimension contains eight items (e.g., "I can always view difficulties from different angles"). The scale is rated on a 5-point Likert scale, ranging from 1 (never) to 5 (always). The total score is considered an indicator of cognitive flexibility. A higher total score indicates higher cognitive flexibility. This scale has been proven to have satisfactory reliability and validity in the Chinese context (Yang et al., 2016). In this study, Cronbach's alpha was 0.90 and the split-half reliability coefficient was 0.87 .

\section{Mental Health}

As suggested by previous studies (Tajima, 2001; Hoge et al., 2004; Hedelin and Jonsson, 2010; Spijker et al., 2010; Harris et al., 2015; Evans-Lacko et al., 2017), this study adopted depression and anxiety as indicators of participants' mental health.

\section{Depression}

A self-rated depression scale (SDS) (Zung, 1965) was used to measure participants' depression. This scale contains 20 items (e.g., "I feel depressed," "I do not sleep well at night," "I do not feel as happy as I did before when I had close contact with the opposite sex") that are rated on a 5-point Likert scale, ranging from 1 (strongly disagree) to 5 (totally agree). Half of the items were scored in reverse. The total score is considered an indicator of depression. The higher the total score, the more severe the depression. In this study, Cronbach's alpha was 0.86 and the split-half reliability coefficient was 0.82 .

\section{Anxiety}

A self-rated anxiety scale (SAS) (Zung, 1971) was used to assess subjective anxiety symptoms. This scale contains 20 items (e.g., "I feel more nervous and anxious than usual," "I feel scared for no reason," and "I get upset or frightened easily") that are rated on a 5 -point Likert scale ranging from 1 (strongly disagree) to 5 (totally agree). Some items were scored in reverse. The total score is considered an indicator of anxiety. The higher the total score, the more severe the anxiety. In this study, Cronbach's alpha was 0.87 and the split-half reliability coefficient was 0.79 .

\section{Demographic Measure}

Participants' demographic information was gathered, including their gender, age, physical health status, family economic status, and level of physical activity.

\section{Statistical Analyses}

SPSS version 22.0 was used for the data analysis. First, a correlation analysis was used to describe the data. Second, a mediation effect test was adopted to explore the mediating effect of cognitive flexibility on the relationship between CINL and mental health, which was indicated by anxiety and depression, respectively.

\section{Results}

\section{Preliminary Analysis}

As shown in Table 5, there was a significant positive correlation between CINL scores and cognitive flexibility, and a significant negative correlation between CINL and depression; however, the correlation between CINL and anxiety was not significant. 
TABLE 5 | Descriptive statistics and correlation matrix for each variable $(N=416)$.

\begin{tabular}{|c|c|c|c|c|c|c|c|c|}
\hline Variable & 1 & 2 & 3 & 4 & 5 & 6 & 7 & 8 \\
\hline 1. Age & 1 & & & & & & & \\
\hline 2. Physical health status & -0.06 & 1 & & & & & & \\
\hline 3. Family financial status & -0.07 & -0.01 & 1 & & & & & \\
\hline 4. Physical activity status & 0.02 & $0.28^{\star \star \star}$ & $0.10^{\star}$ & 1 & & & & \\
\hline 5. CINL & -0.04 & -0.05 & $0.20^{\star \star \star}$ & $0.14^{\star \star}$ & 1 & & & \\
\hline 6. Cognitive flexibility & 0.05 & $0.19^{\star \star \star}$ & $0.13^{\star \star}$ & $0.25^{\star \star \star}$ & $0.16^{\star \star}$ & 1 & & \\
\hline 7. Anxiety & 0.06 & $-0.22^{\star \star \star}$ & $-0.20^{\star}$ & $-0.13^{\star \star}$ & -0.07 & $-0.44^{\star \star \star}$ & 1 & \\
\hline 8. Depression & 0.04 & $-0.19^{\star \star \star}$ & $-0.22^{\star \star \star}$ & $-0.19^{\star \star \star}$ & $-0.17^{\star \star \star}$ & $-0.43^{\star \star \star}$ & $0.76^{\star \star \star}$ & 1 \\
\hline M & 19.83 & 1.97 & 2.79 & 2.27 & 17.32 & 66.85 & 48.80 & 50.79 \\
\hline$S D$ & 1.38 & 0.43 & 0.59 & 0.66 & 3.73 & 8.97 & 10.56 & 9.64 \\
\hline
\end{tabular}

${ }^{*} p<0.05,{ }^{* *} p<0.01,{ }^{* * *} p<0.001$.

Cognitive flexibility was negatively correlated with anxiety and depression. A significant positive correlation was found between anxiety and depression.

\section{Relationship Between CINL and Mental Health: Test Model for the Mediating Effect of Cognitive Flexibility} The PROCESS of SPSS macro program model 4 was used to explore the mediating effect of cognitive flexibility on the relationship between CINL and depression, with CINL as an independent variable, cognitive flexibility as an intermediary variable, and depression as the dependent variable. Age, gender, family economic status, physical health status, and physical activity status were used as control variables.

As shown in Figure 1, after controlling for gender, age, family economic status, physical health status, and physical activity status, CINL was found to be significantly related to cognitive flexibility, and cognitive flexibility was significantly related to depression. However, the effect of CINL on depression was not significant in this model. The Sobel test showed that the mediation model was significant $(B=-0.10$, $\mathrm{SE}=0.006$, $z=-2.72, p<0.05)$. The boost SE 95\% confidence interval was $[-0.2232,-0.0014]$ and did not include 0 . Furthermore, the ratio of the indirect effect to the total effect was $36.93 \%$. This suggests that cognitive flexibility fully mediates the relationship between CINL and anxiety.

Similarly, the PROCESS of SPSS macro program model 4 was used to explore the mediating effect of cognitive flexibility on the relationship between CINL and anxiety. This study used CINL as an independent variable, cognitive flexibility as an intermediary variable, and anxiety as the dependent variable, while age, gender, family economic status, physical health status, and physical activity status were set as control variables.

As shown in Figure 2, after controlling for gender, age, family economic status, physical health status, and physical activity status, CINL significantly predicted cognitive flexibility, and cognitive flexibility was a significantly negative predictor of anxiety. The Sobel test showed that the mediation model was significant $(B=-0.12, \mathrm{SE}=0.006, z=-3.08, p<0.05)$. The boost SE 95\% confidence interval was $[-0.2232,-0.0014]$ and did not include 0 . This suggests that cognitive flexibility fully mediates the relationship between CINL and anxiety.
In a word, cognitive flexibility played a full but not partly mediating role in CINL and mental health, which partially supported the hypothesis.

\section{DISCUSSION}

This study explored the effects of CINL on mental health. The results showed that CINL improved mental health by promoting cognitive flexibility, which supports our hypothesis. These results are of great theoretical and practical significance in understanding the mechanisms of cyber behavior and mental health.

With regard to the demographic variables, the results of this research are partially consistent with those of previous studies on physical health status (Dong et al., 2012; Lu and Wang, 2015), family economic status (Hudson, 2005; Hu et al., 2014), and physical activity status (Swann et al., 2018; Zheng et al., 2020), with a significant positive relationship with mental health. In addition, the differences in mental health between genders were found to be marginally significant in this study, which is consistent with previous studies that have shown mental health of women was slightly lower than that of men (Zhang and Yu, 2004; Feng and Zhang, 2005; He, 2014). With regard to age, the study did not find a significant relationship between age and mental health, which differs from a previous study (Zhang et al., 2011). This may be due to the limited age range included in this study.

As expected, this study found that CINL was significantly and positively correlated with cognitive flexibility, and negatively correlated with depression. However, CINL was not significantly associated with anxiety. After controlling for cognitive flexibility, CINL was shown to have a positive effect on anxiety, although this was still not significant and somewhat different from the hypothesis. This deviation may be due to the fact that CINL may inadvertently trigger an individual's panic and anxiety toward the COVID-19 epidemic. Previous studies have demonstrated that when facing stressors, individuals produce corresponding stress responses, such as symptoms related to an excited sympathetic nervous system, high secretion of pituitary and adrenal corticosteroids, and accelerated respiration (McGraw et al., 2013). These physiological responses are easily associated 


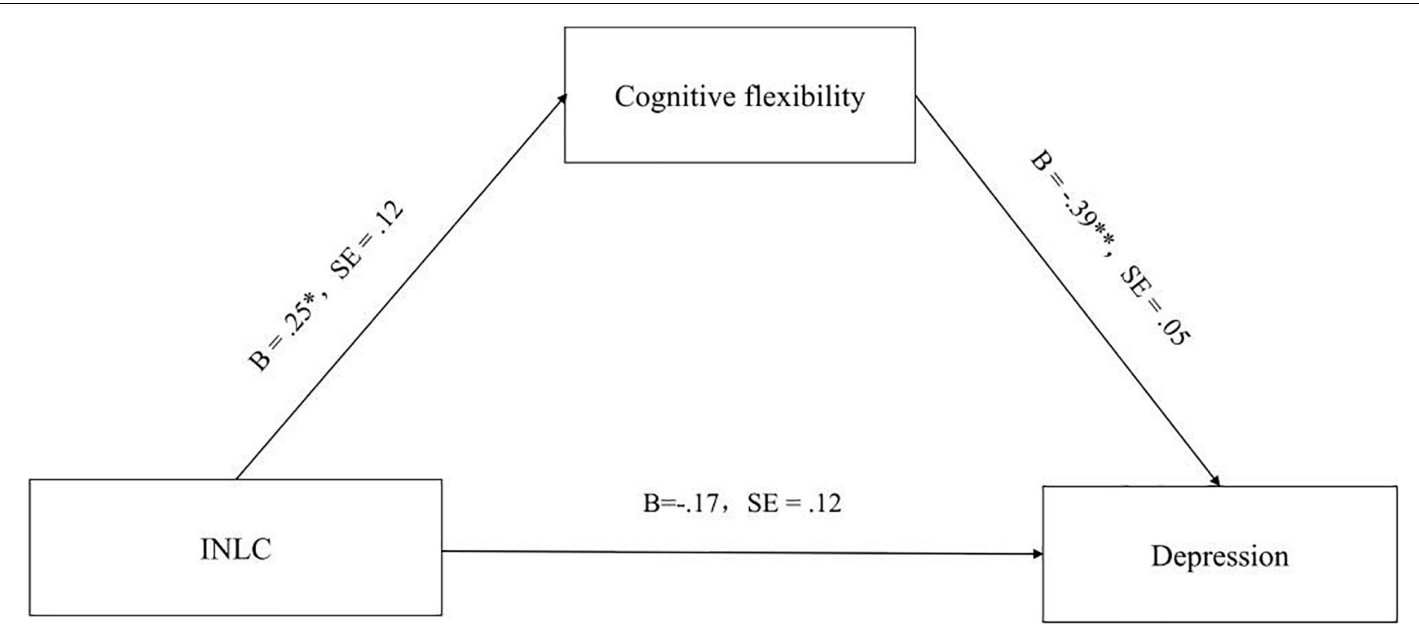

FIGURE 1 | The mediating of effect of cognitive flexibility on the link between CINL and depression in the entire sample.

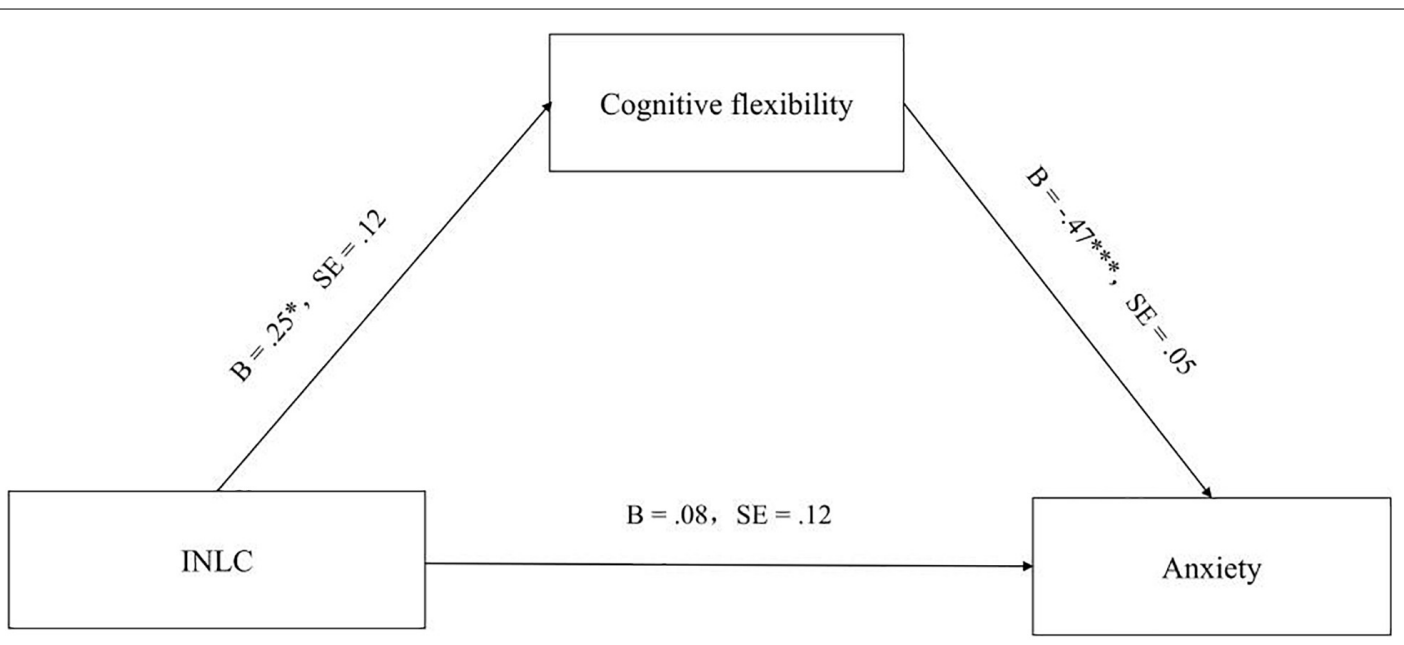

FIGURE 2 | The mediating of effect of cognitive flexibility on the link between CINL and anxiety.

with anxiety. From this perspective, CINL's impact on anxiety may be masked by the inhibiting effect of CINL mediated through cognitive flexibility, which may also mask the effect of exposure to CINL on anxiety. Therefore, the overall effect of CINL on anxiety was not significant.

This study hypothesized that cognitive flexibility plays a partial mediating role between CINL and mental health; however, the results showed that cognitive flexibility played a fully mediating effect. This may be because positive emotions, which are triggered by CINL, can promote individuals' cognitive flexibility (Liang and Chen, 2015; Wang et al., 2017). Therefore, cognitive flexibility plays a full mediating role between CINL and mental health.

Previous studies on mental health have focused on cognitive factors such as cognitive flexibility, creativity, and coping strategies (Li, 2003; Luo and Lin, 2006; Wei and Fu, 2007), as well as social factors such as social support, social adaptation, and stress events (Wen et al., 2000; Ding et al., 2005; Tu and Guo, 2010). However, few studies have combined two or more of these factors to examine them simultaneously. Nowadays, the development of information is rapid; the Internet as a new medium of information communication and as an interpersonal communication tool is changing modern life and it is becoming increasingly important in people's studies, work, life, and mental health (Chen, 2001).

Internet language lies at the core of Internet information transmission, and may have an effect as a result of a combination of factors. First, CINL is updated rapidly and is closely related to social events, requires individuals to be knowledgeable and have their own understanding of social events, and can undoubtedly increase the social adaptability of individuals. In the process of conducting the survey, we found that people with different social statuses and from demographic categories use different INLs. When the INL that an individual uses is commonly used in his/her groups, this may improve the individual's social identity and sense of belonging, thus promoting individual social support, which is important for mental health (Pang, 2009; 
Kong et al., 2010). These studies suggest that INL not only plays an important role on the Internet, but also covers all aspects of real life and impacts more than just mental health. However, INL has not yet become a focus of the majority of researchers. This study preliminarily explores the relationship between INL and mental health while considering the mediating effect of cognitive flexibility, which provides a direction for future research on INL.

In addition, this study also has practical significance for interventions regarding college students' mental health. On the one hand, we can promote the development of college students' mental health through their preferred INL. On the other hand, due to the adverse effects of Internet use, some people, especially parents, are afraid of too much exposure to the Internet (Lei et al., 2012; Kuss et al., 2013; Lam, 2014; Yeh and Cheng, 2016; Cheung et al., 2018). They are worried that their children's exposure to the Internet will affect their physical and mental health development. According to the results of this study, it is advisable to take an inclusive attitude toward the Internet.

This study had several limitations. First, this study used a cross-sectional paradigm; therefore, a causal relationship could not be established. Thus, future studies should adopt longitudinal cross-sectional studies or experimental methods to improve internal validity. Second, the study used a self-report method, which might not accurately indicate the use of CINL and mental health conditions. Thus, future studies should adopt more accurate indicators, such as big data analysis of CINL and objective physiological measurements of mental health. Finally, this study discusses the effect of INL on mental health in general; however, the effects of different types of INL on mental health may vary, which warrants further discussion.

\section{CONCLUSION}

In the context of the COVID-19 pandemic, CINL could alleviate individuals' depression and anxiety through the mediating pathway of cognitive flexibility. Given that most of the previous research on cyber behavior has focused on its negative effects, the results of this study provide a positive perspective on understanding the effects of cyber behavior on human mental health that may help researchers to gain a more comprehensive insight into cyber behaviors. Furthermore, INL is easy and convenient to use in daily life, so it also provides practitioners with guidance for mental health interventions.

\section{REFERENCES}

Al-Mosaiwi, M., and Johnstone, T. (2018). In an absolute state: elevated use of absolutist words is a marker specific to anxiety, depression and suicidal ideation. Clin. Psychol. Sci. 6, 529-542. doi: 10.1177/216770261774 7074

Angoff, W. H. (1953). Test reliability and effective test length. Psychometrika 18, 1-14. doi: $10.1007 / \mathrm{bf} 02289023$

Barceló, F., Periáez, J. A., and Knight, R. T. (2002). Think differently: a brain orienting response to task novelty. NeuroReport 13, 1887-1892. doi: 10.1097/ 00001756-200210280-00011

Brewster, M. E., Moradi, B., Deblaere, C., and Velez, B. L. (2013). Navigating the borderlands: the roles of minority stressors, bicultural

\section{DATA AVAILABILITY STATEMENT}

The raw data supporting the conclusions of this article will be made available by the authors, without undue reservation.

\section{ETHICS STATEMENT}

The studies involving human participants were reviewed and approved by the School of Psychology, Central China Normal University. The patients/participants provided their written informed consent to participate in this study.

\section{AUTHOR CONTRIBUTIONS}

ZW and XW: investigation and writing the original draft. QY, $\mathrm{ZZ}$, and QZ: conceptualization, formal analysis, investigation, writing original draft, and project administration. HZ and PY: methodology, review writing, and editing. XW and QY: revised the manuscript. All authors approved the final version of the manuscript for submission.

\section{FUNDING}

This research was supported by a grant from the Research Program Funds of the Collaborative Innovation Center of Assessment Toward Basic Education Quality (2019-04020BZPK01), research funding from the Key Laboratory of Adolescent Cyberpsychology and Behavior (CCNU), Ministry of Education (2018B01), a grant from the Postdoctoral Science Foundation of China (2019M652679), a grant from the Fundamental Research Funds for the Central Universities (CCNU18ZDPY07), and a grant from the self-determined research funds of the CCNU from the colleges' basic research and operation of MOE (CCNU19TD018).

\section{ACKNOWLEDGMENTS}

We would like to thank Editage (www.editage.cn) for English language editing.

self-efficacy, and cognitive flexibility in the mental health of bisexual individuals. J. Couns. Psychol. 60, 543-556. doi: 10.1037/a003 3224

Canas, J., Quesada, J. F., Antoli, A., and Fajardo, I. (2003). Cognitive flexibility and adaptability to environmental changes in dynamic complex problemsolving tasks. Ergonomics 46, 482-501. doi: 10.1080/001401303100006 1640

Chen, Z. (2001). Computer network and the psychological health of the network users. Stud. Dialectics Nat. 17, 67-71.

Cheung, J. C., Chan, K. H., Lui, Y., Tsui, M., and Chan, C. (2018). Psychological well-being and adolescents' internet addiction: a school-based cross-sectional study in Hong Kong. Child Adolesc. Soc. Work J. 35, 477-487. doi: 10.1007/ s10560-018-0543-7 
Dennis, J. P., and Wal, J. S. V. (2010). The cognitive flexibility inventory: instrument development and estimates of reliability and validity. Cogn. Therapy Res. 34, 241-253. doi: 10.1007/s10608-009-9276-4

Ding, Y., Xiao, L., and Guo, W. (2005). Working model of social support in relationship of life events and mental health. Chin. J. Health Psychol. 13, 161-164.

Dong, Y., Wang, Q., and Xing, C. (2012). Advances in the study of the relationship between positive emotion and health. J. Psychol. Sci. 35, 487-493.

$\mathrm{Du}, \mathrm{L}$. (2007). A brief analysis of the network language patterns, causes and disputes. Read Write Periodical 4, 120-121.

Edwards, D. J. (2019). Age, pain intensity, values-discrepancy, and mindfulness as predictors for mental health and cognitive fusion: hierarchical regressions with mediation analysis. Front. Psychol. 10:517.

Evans-Lacko, S., Aguilar-Gaxiola, S., Al-Hamzawi, A., Alonso, J., Benjet, C., and Bruffaerts, R. (2017). Socio-economic variations in the mental health treatment gap for people with anxiety, mood, and substance use disorders: results from the who world mental health (wmh)\\udsurveys. Psychol. Med. 48, 1560-1571.

Feng, Z., and Zhang, D. (2005). Dliferences of midtile school students'depressive symptoms development in gender,age and grade. Chin. J. Clin. Rehabil. 9, 32-34.

Friedland, D. L., and Michael, W. B. (1987). The reliability of a promotional job knowledge examination scored by numberof items right and by four confidence weighting procedures and its corresponding concurrent validity estimates relative to performance criterion ratings. Educ. Psychol. Meas. 47, 179-188. doi: 10.1177/0013164487471026

Guo, X., and Wang, Z. (2007). Concept, function and meaning of positive emotion. Adv. Psychol. Sci. 15, 810-815.

Harris, M. G., Hobbs, M. J., Burgess, P. M., Pirkis, J. E., Diminic, S., and Siskind, D. J. (2015). Frequency and quality of mental health treatment for affective and anxiety disorders among australian adults. Med. J. Aust. 202, 185-189. doi: $10.5694 / \mathrm{mja} 14.00297$

Hasselbalch, B., Knorr, U., Hasselbalch, S., Gade, A., and Kessing, L. (2012). Cognitive deficits in the remitted state of unipolar depressive disorder. Neuropsychology 25, 642-651. doi: 10.1037/a0029301

He, E. (2014). Gender difference of university students in metal health, personality and sense of inferiority. Educ. Teaching Res. 28, 44-46.

Hedelin, B., and Jonsson, I. (2010). Mutuality as background music in women's lived experience of mental health and depression. J. Psychiatr. Ment. Health Nurs. 10, 317-322. doi: 10.1111/1540-5885.310001-i1

Hellman, C. M., Fuqua, D. R., and Worley, J. (2006). A reliability generalization study on the survey of perceived organizational support: the effects of mean age and number of items on score reliability. Educ. Psychol. Meas. 66, 631-642. doi: $10.1177 / 0013164406288158$

Hoge, C. W., Castro, C. A., Messer, S. C., McGurk, D., Cotting, D. I., and Koffman, R. L. (2004). Combat duty in Iraq and Afghanistan, mental health problems, and barriers to care. N. Engl. J. Med. 351, 13-22. doi: 10.1056/nejmoa 040603

$\mathrm{Hu}, \mathrm{X} ., \mathrm{Li}, \mathrm{J} ., \mathrm{Lu}, \mathrm{X}$., and Guo, Y. (2014). The psychological study of social class: social cognitive perspective. J. Psychol. Sci. 34, 1509-1517.

Huang, F., Zhou, Z., and Zhao, Q. (2013). An eye movement study of associate competition in Chinese idiom riddles solving. Acta Psychologica Sinica 45, 35-46. doi: 10.3724/sp.j.1041.2013.00035

Hudson, C. G. (2005). Socioeconomic status and mental illness: tests of the social causation and selection hypotheses. Am. J. Orthopsychiatry 75, 3-18. doi: 10. 1037/0002-9432.75.1.3

Jahja, R., Van Spronsen, F. J., De Sonneville, L. M. J., Van der Meere, J. J., Bosch, A. M., and Hollak, C. E. M. (2017). Long-term follow-up of cognition and mental health in adult phenylketonuria: a pku-cobeso study. Behav. Genet. 47, $1-12$.

Jen, H. C., Chen, W., and Wu, C. (2018). Flexible mindset in the family: filial piety, cognitive flexibility, and general mental health. J. Soc. Pers. Relat. 36, 1715-1730. doi: $10.1177 / 0265407518770912$

Jowkar-Baniani, G., and Schmuckler, M. A. (2014). The role of stimulus novelty on children's inflexible dimensional switching. Child Dev. 85, 1373-1384. doi: $10.1111 /$ cdev. 12212

Kim, B. S. K., and Omizo, M. M. (2005). Asian and European American cultural values, collective self-esteem, acculturative stress, cognitive flexibility, and general self-efficacy among Asian American college students. J. Couns. Psychol. 52, 412-419. doi: 10.1037/0022-0167.52.3.412
Koesten, J., Schrodt, P., and Ford, D. J. (2009). Cognitive flexibility as a mediator of family communication environments and young adults' well-being. Health Commun. 24, 82-94. doi: 10.1080/10410230802607024

Kong, L., Jiang, X., and Ji, C. (2010). Study on relationship between social support and sense of school belonging of middle school students. China J. Health Psychol. 18, 451-453.

Kuss, D. J., van Rooij, A. J., Shorter, G. W., Griffiths, M. D., and van de Mheen, D. (2013). Internet addiction in adolescents: prevalence and risk factors. Comput. Hum. Behav. 29, 1987-1996.

Lam, L. T. (2014). Risk factors of Internet addiction and the health effect of internet addiction on adolescents: a systematic review of longitudinal and prospective studies. Curr. Psychiatry Rep. 16:508.

Lei, P., Feng, D., and Tan, X. (2012). Development and preliminary application of adolescents online stigmatization. Chinese J. Clin. Psychol. 20, 328-331.

Li, W. (2003). Mental health: definition and feathers. Educ. Res. 10, 69-75.

Liang, J., and Chen, S. (2015). How positive emotion affects cognition:a review on the development of models. Chinese J. Appl. Psychol. 21, 157-165.

Liu, S., Bi, X., and He, K. (2017). The impact of Internet language copy on consumers' attention and perceptions of the advertisement. Acta Psychologica Sinica 49, 1590-1603. doi: 10.3724/sp.j.1041.2017.01590

Liu, S., Chen, P., and Ding, G. (2019). Product involvement, language attitude and theeffectiveness of ads with Internet slang. J. Psychol. Sci. 42, 124-129.

Lord, F. M., and Novick, M. R. (1968). Statistical Theories of Mental Test Scores. Boston, MA: Addison-Wesley Educational Publishers.

Lu, S., and Wang, D. (2015). Mental health conditions and its influential factors among officers and soldiers in army troop. Chin. Prev. Med. 16, 708-711.

Luo, X., and Lin, C. (2006). Construct the model of multi-layer relations among mental health, personality and creativity of Chinese university students. J. Psychol. Sci. 29, 1031-1034.

Ma, W., Li, Y., Luo, J., and Zhang, Q. (2012). Competition and emotion impact on effect of prototype elicitation during insight problem solving. Acta Psychologica Sinica 44, 1-13. doi: 10.3724/sp.j.1041.2012.00001

McGraw, L. K., Out, D., Hammermeister, J. J., Ohlson, C. J., Pickering, M. A., and Granger, D. A. (2013). Nature, correlates, and consequences of stress-related biological reactivity and regulation in army nurses during combat casualty simulation. Psychoneuroendocrinology 38, 135-144. doi: 10.1016/j.psyneuen. 2012.05.009

Mehri, L., and Bakhtiarpoor, S. (2016). Investigating the coping relationship of problem-oriented and excitement-oriented and cognitive flexibility with mental health. Eur. Online J. Nat. Soc. Sci. 6, 535-545.

Murphy, F., Michael, A., and Sahakian, B. (2012). Emotion modulates cognitive flexibility in patients with major depression. Psychol. Med. 42, 1373-1382. doi: $10.1017 / \mathrm{s} 0033291711002418$

Odacı, H., and Çikrikci, Ö (2018). Cognitive flexibility mediates the relationship between big five personality traits and life satisfaction. Appl. Res. Qual. Life 14, 1229-1246. doi: 10.1007/s11482-018-9651-y

Ornell, F., Schuch, J. B., Sordi, A. O., and Kessler, F. H. P. (2020). "Pandemic fear" and COVID-19: mental health burden and strategies. Braz. J. Psychiatry 43, 232-235. doi: 10.1590/1516-4446-2020-0008

Pang, H. (2009). A Research on the relationship between the school belonging and mental health of junior middle school sudents. J. Psychol. Sci. 32, 12381241.

Perpiñá, C., Segura, M., and Sánchez-Reales, S. (2016). Cognitive flexibility and decision-making in eating disorders and obesity. Eat. Wght Disord. 22, 435-444. doi: 10.1007/s40519-016-0331-3

Shen, W., Yuan, Y., Gong, Z., and Liu, C. (2017). The property, structure, and functional base of insight experience. J. Psychol. Sci. 40, 347-352.

Spijker, J., Bijl, R. V., Graaf, R. D., and Nolen, W. A. (2010). Determinants of poor 1 -year outcome of dsm-iii-r major depression in the general population: results of the netherlands mental health survey and incidence study (nemesis). Acta Psychiatr. Scand. 103, 122-130. doi: 10.1034/j.1600-0447.2001.103002122.x

Swann, C., Telenta, J., Draper, G., Liddle, S., Fogarty, A., and Hurley, D. (2018). Youth sport as a context for supporting mental health: adolescent male perspectives. Psychol. Sport Exerc. 33, 55-64. doi: 10.1016/j.psychsport.2017. 11.008

Tajima, O. (2001). Mental health care in japan: recognition and treatment of depression and anxiety disorders. J. Clin. Psychiatry 62, 39-44. 
Tchanturia, K., Anderluh, M., Morris, R., Rabe-Hesketh, S., Collier, D., Sanchez, P., et al. (2004). Cognitive flexibility in anorexia nervosa and bulimia nervosa. J. Int. Neuropsychol. Soc. 10, 513-520.

Tu, Y., and Guo, Y. (2010). Posttraumatic growth: conception, influential factors and relationships with mental health. Adv. Psychol. Sci. 18, 114-122.

Vahia, I. V., Blazer, D. G., Smith, G. S., Karp, J. F., Steffens, D. C., Forester, B. P., et al. (2020). COVID-19, mental health and aging: a need for new knowledge to Bridge science and service. Am. J. Geriatr. Psychiatry 28, 695-697. doi: 10.1016/j.jagp.2020.03.007

Wang, M., Cao, R., Zhang, L., Yang, X., Liu, J., Xu, M., et al. (2020). Remdesivir and chloroquine effectively inhibit the recently emerged novel coronavirus (2019-nCoV) in vitro. Cell Res. 30, 269-271. doi: 10.1038/s41422-020-0282-0

Wang, Y., Yang, Y., Xiao, W., and Qin, S. (2016). Validity and reliability of the Chinese version of the cognitive flexibility inventory in college students. Chin. Ment. Health J. 30, 58-63.

Wang, Y., Jie, C., and Yue, Z. (2017). Positive emotion facilitates cognitive flexibility: an fmri study. Front. Psychol. 8:1832.

Wei, M., and Fu, F. (2007). Relationship between emotional regulation and mental health of college students. China J. Health Psychol. 15, 108-111.

Wen, W., Wang, Y., and Sun, J. (2000). Research on the relationship between social support, psychological control and psychological health. Chin. Ment. Health J. 14, 258-260.

Windle, M. (2010). Sex role orientation, cognitive flexibility, and life satisfaction among older adults. Psychol. Women Q. 10, 263-273. doi: 10.1111/j.1471-6402. 1986.tb00752.x

World Health Organization (2021). WHO Coronavirus (COVID-19) Dashboard | WHO Coronavirus (COVID-19) Dashboard with Vaccination Data. Available online at: https://covid19.who.int/

$\mathrm{Wu}, \mathrm{X}$. (2004). The contextual features of network and the semantic means of network language. ChangChun Univer. Sci. Technol. 17, 12-14.

$\mathrm{Xu}, \mathrm{M}$. (2015). Construction principle and understanding mechanism of irrelevant homophony in internet language. Contemporary Rhetoric 6, 54-63.

Yang, Y., and Li, L. (2007). The relationship between adolescents' extraversion agreeableness, internet service preference, and Internet addiction. Psychol. Dev. Educ. 2, 42-48.

Yang, Y., Li, L., and Liu, M. (2006). The relationship between adolescents' conscientiousness, internet service preference and internet addiction. J. Psychol. Sci. 29, 947-950.

Yang, Y., Wang, Y., Xiao, W., and Qin, S. (2016). Validity and reliability of the Chinese version of the cognitive flexibility inventory in college students. Chin. Ment. Health J. 30, 58-63.

Yao, Z., and Zhou, M. (2019). Influence of internet language on mental health education of college students. Sci. Technol. Inform. 5, 183-184.
Yeh, D. Y., and Cheng, C. H. (2016). Relationships among Taiwanese children's computer game use, academic achievement and parental governing approach. Res. Educ. 95, 44-60. doi: 10.7227/rie.0025

Yu, Q., Zhang, L., Wu, S., Guo, Y., Jin, S., and Sun, Y. (2017). The influence of juvenile preference for online social interaction on problematic internet use: the moderating effect of sibling condition and the moderated moderating effect of age cohort. Comput. Hum. Behav. 68, 345-351. doi: 10.1016/j.chb.2016. 11.026

Zhang, W., Guo, F., and Chen, Z. (2011). Relationship of stress, optimism, social support and mental health. Chin. J. Clin. Psychol. 19, 225-227.

Zhang, Y., and Yu, G. (2004). Mental health in pre-adolescence: development trends and gender differences. Chin. J. Clin. Psychol. 12, 380-382.

Zhang, Y., Chen, S., Li, S., Zhao, Q., Zhou, Z., Huang, F., et al. (2019). The use of internet language enhances creative performance. J. Gen. Psychol. 148, 26-44. doi: 10.1080/00221309.2019.1703628

Zhao, J., Luo, Z., and Wang, X. (2010). The relationship between mental resilience, positive emotion and mental health of college graduates. China J. Health Psychol. 18, 1078-1080.

Zhao, Q., Ke, W., Tong, B., Zhou, Z., and Zhou, Z. (2017). Creative processing of Internet language: Novel N400 and LPC. Acta Psychologica Sinica 49, 143-154. doi: 10.3724/sp.j.1041.2017.00143

Zheng, X., Chen, J., Guo, Y., Qing, X., Hu, Y., Shi, S., et al. (2020). The buffer effect of physical activity: why does parental marital satisfaction affect adolescents' problematic Internet use. Addict. Behav. Rep. 11:100271. doi: 10.1016/j.abrep. 2020.100271

Zhou, M. (2010). Cognitive research on chinese internet language. J. Chifeng Univer. 31, 113-114.

Zung, W. W. (1965). A self-rating depression scale. Arch. Gen. Psychiatry 12, 63-70.

Zung, W. W. (1971). A rating instrument for anxiety disorders. Psychosomatics 12, 371-379. doi: 10.1016/s0033-3182(71)71479-0

Conflict of Interest: The authors declare that the research was conducted in the absence of any commercial or financial relationships that could be construed as a potential conflict of interest.

Copyright (c) $2021 \mathrm{Wu}$, Wang, Zhang, Yuan, Yu, Zhou and Zhao. This is an openaccess article distributed under the terms of the Creative Commons Attribution License (CC BY). The use, distribution or reproduction in other forums is permitted, provided the original author(s) and the copyright owner(s) are credited and that the original publication in this journal is cited, in accordance with accepted academic practice. No use, distribution or reproduction is permitted which does not comply with these terms. 